\title{
APRENDER EM PROJETOS DE INVESTIGAÇÃO DURANTE A LICENCIATURA EM ENFERMAGEM: REVISÃO INTEGRATIVA DA LITERATURA
}

\author{
David Loura', Rafael Bernardes ${ }^{2}$, Cristina Lavareda Baixinho ${ }^{1,3,4}$, Helga Rafael ${ }^{1,3}$, Isa \\ Félix $^{1}$, Mara Guerreiro ${ }^{1,3,5}$ \\ ${ }^{1}$ Escola Superior de Enfermagem de Lisboa, Portugal. davidloura@campus.esel.pt isafelix@esel,pt \\ ${ }^{2}$ UICISA:E, Escola Superior de Enfermagem de Coimbra, Coimbra, Portugal rafaelalvesbernardes@esenfc.pt \\ ${ }^{3}$ UIDE, Portugal. hrafael@esel,pt \\ ${ }^{4}$ ciTechCare, Portugal. crbaixinho@esel.pt \\ ${ }^{5}$ CiiEM, Instituto Universitário Egas Moniz, Monte de Caparica, Portugal. mara.guerreiro@esel.pt
}

\begin{abstract}
Resumo. Introdução: O desenvolvimento da investigação e a emergência de uma prática baseada na evidência são um desafio claro para a educação dos futuros profissionais de saúde, aos quais são requeridas competências de pesquisa, leitura e utilização correta dos resultados da investigação na prática clínica. Objetivos: Identificar o que aprendem os estudantes de enfermagem com o envolvimento em projetos de investigação, durante a licenciatura. Métodos: A revisão integrativa de literatura foi efetuada segundo um protocolo, com definição dos critérios de elegibilidade dos estudos primários, obtidos nas bases de dados dos motores de busca EBSCO, $\mathrm{JBI}$ e Scopus. Resultados: Os estudantes podem ser envolvidos em todas as fases do processo de pesquisa, contribuindo para a sua satisfação na aprendizagem, com impacto positivo na motivação, reflexão e integração do conhecimento, desenvolvimento de competências de comunicação, escrita científica, gestão do tempo e juízo critico. $O$ envolvimento dos estudantes contribui para o desenvolvimento dos projetos de investigação. Conclusões: O envolvimento dos estudantes em projetos de investigação contribui para diferentes aprendizagens. Os nossos resultados sugerem que a formação teórica sobre investigação deve ser associada a outras estratégias para o desenvolvimento de conhecimentos, habilidades e atitudes de pesquisa.
\end{abstract}

Palavras-chave: Estudantes; Enfermagem; Pesquisa; Aprendizagem.

LEARNING IN RESEARCH PROJECTS DURING NURSING UNDERGRADUATE DEGREE: INTEGRATIVE LITERATURE REVIEW

Abstract. Introduction: The development of research and the evidence-based practice paradigm pose a challenge for the education of future health professionals, who are required to have search skills, and be able to appraise and correctly use research results in clinical practice. Objectives: To identify what nursing students learn from being involved in research projects during their undergraduate degree. Methods: This integrative literature review was carried out according to a protocol, defining the eligibility criteria for primary studies, obtained from EBSCO, JBI and Scopus search engines. Results: Students can be involved in all phases of the research process, contributing to their satisfaction with learning, with positive impact on motivation, reflection and integration of knowledge, development of communication skills, scientific writing, time management and critical judgment. Involving these students also benefits the research process. Conclusions: Students' involvement in research projects contributes to a range of learning opportunities. Our results suggest that classroom education on research should be associated with other strategies for the development of research knowledge, skills and attitudes.

Keywords: Students; Nursing; Research; Learning. 


\section{INTRODUÇÃO}

Há um interesse crescente sobre a pesquisa e o desenvolvimento da investigação em saúde e o envolvimento de estudantes da formação pré e pós graduada, com recomendações para que os futuros profissionais de saúde sejam envolvidos neste tipo de atividades, a fim de nutrir uma cultura e um espírito de pesquisa (Einarsen \& Giske, 2019). Associado a este movimento tem emergido nos currículos em enfermagem a introdução de educação em Prática Baseada em Evidência (PBE), para o desenvolvimento académico dos estudantes e aprofundamento dos conteúdos teóricos (Mena-Tudela et al., 2018). Tal promove uma integração teórico-prática, essencialmente, durante o ensino clinico para que este tenha um impacto na melhoria dos cuidados e facilite o desenvolvimento de um novo profissional de saúde, com competências científicas de diferentes níveis, apto para reger a sua atividade profissional pelos resultados da investigação.

É inegável que os cuidados de saúde estão a tornar-se mais complexos, exigindo um aumento de competências científicas (Einarsen \& Giske, 2019). Todavia, é difícil alcançar os objetivos referidos de um modo tradicional em que um professor prepara aulas, explica e demonstra aos seus estudantes (Sun, Liu, Wu, \& Li, 2014), esperando que desta, forma este, saiba utilizar a pesquisa para produzir resultados seguros na clínica. A educação em enfermagem deve fortalecer a capacidade de pensamento crítico e aprimorar as habilidades dos estudantes nas áreas de pensamento analítico, resolução de problemas e raciocínio clínico (Einarsen \& Giske, 2019). Tais habilidades proporcionam um maior desenvolvimento profissional, facilitam a compreensão de situações mais complexas nos processos de saúde/doença das pessoas e prepara os enfermeiros para um escopo mais amplo de prática (Einarsen \& Giske, 2019).

Slattery et al. (2016) defendem que para além de aprender teoria sobre investigação, familiarizar-se com trabalho interprofissional a este nível, importa que os estudantes participem em pesquisa, interajam efetivamente com investigadores académicos e clínicos e incorporarem os resultados da investigação nas intervenções de enfermagem, obtendo competências de PBE e a melhoria da qualidade dos cuidados de saúde (Baixinho, Ferreira, Marques, Presado, \& Cardoso 2017).

Alguns autores advogam que os Ensinos Clínicos são momentos-chave para a melhoria dos conhecimentos, atitudes e competências dos estudantes ao nível da evidência científica (Aglen, 2016; Mena-Tudela et al., 2018), considerando que a aprendizagem de uma PBE é 
maior quando é integrada no ensino clínico, provavelmente pela possibilidade de experienciar o processo e o impacto na melhoria dos cuidados nos diferentes contextos de práxis.

A aprendizagem de uma PBE não se aprende nos bancos da escola (Slattery et al., 2016; Mena-Tudela et al., 2018), exige um contacto com projetos de investigação, conhecimento de métodos e técnicas de investigação e capacidade de análise sobre o desenho do estudo, a sua qualidade e os resultados obtidos, ou seja, que o estudante seja um participante ativo em alguma fase do processo de pesquisa.

Os estudos sobre o tema registam as vantagens, mas não são claros quanto ao impacto destas experiências após o seu término. Na avaliação do programa de iniciação à investigação desenvolvido por Slattery et al. (2016) os resultados apontam para que a exposição precoce, dos estudantes de enfermagem, a diferentes atividades de pesquisa encoraje os recém-formados a incorporar a evidência na sua clínica futura e a serem mais proativos na procura de formação pós-graduada. No entanto, Allari (2016) observa que cerca de $50 \%$ dos estudantes que são envolvidos em experiências de pesquisa durante o curso consideram que a mesma foi difícil e mesmo vendo os resultados aplicados na prática não manifestam intenção de realizar investigação no futuro.

Foster e Flanders (2014) na investigação que efetuaram sobre os desafios da educação de enfermeiros também alertam para existência de barreiras à aprendizagem de uma PBE, tais como as descrições pouco claras das atividades efetuadas pelos enfermeiros, dado que muitas vezes em vez de lidar com as atividades especificas da profissão, estão a resolver os problemas periféricos dos doentes, com sobrecarga de trabalho, escassez de tempo, o que impede o uso da evidência clínica na educação dos estudantes (Foster \& Fladers, 2014), e na prestação de cuidados à pessoa hospitalizada. E estas dificuldades na clínica podem ter influência no envolvimento dos estudantes na investigação, na percepção do que é uma PBE e de como pode ser aplicada na clínica.

Independentemente das dificuldades a educação surge como um fator facilitador da integração da PBE na prática clínica (Cardoso et al., 2019) e da aprendizagem de investigação. Uma vez que estes futuros profissionais precisam de ter competências de investigação e de utilização dos resultados da mesma para terem uma PBE é objetivo deste estudo: identificar o que aprendem os estudantes de enfermagem com o envolvimento em projetos de investigação, durante a licenciatura. 


\section{METODOLOGIA}

A presente Revisão Integrativa da Literatura $(R I L)$ pretende responder à seguinte questão de pesquisa, formulada em consonância com a mnemónica PICo (população, fenómeno de interesse e contexto): "O que aprendem os estudantes de enfermagem com 0 envolvimento/participação em projetos de investigação durante o curso de licenciatura?".

A opção pelo método deve-se à possibilidade de incluir literatura relevante que suporte a tomada de decisão, através da síntese do estado do conhecimento sobre o fenómeno em estudo, além de apontar lacunas do conhecimento que precisam de ser preenchidas com a realização de novos estudos (Mendes, Silveira, \& Galvão, 2008).

Para aumentar o rigor do estudo e garantir a validade dos resultados optou-se por seguir o protocolo definido por Mendes, Silveira e Galvão (2008), para este tipo de revisão da literatura com método científico e que compreende seis etapas: (1) identificação do tema e seleção da hipótese ou questão de pesquisa, (2) estabelecimento de critérios para inclusão e exclusão de estudos, (3) definição das informações a serem extraídas, (4) avaliação dos estudos incluídos, (5) interpretação dos resultados e (6) apresentação da revisão/síntese do conhecimento.

Esta orientação metodológica facilitou a seleção dos critérios de inclusão e exclusão dos estudos, com a finalidade de estreitar os intervalos de confiança, facilitar a comparação dos trabalhos, interpretação dos dados e aumentar a precisão dos resultados (Quadro 1).

Quadro 1. Critérios de elegibilidade dos artigos. Lisboa, 2020.

\begin{tabular}{|c|c|c|}
\hline & Critérios de Inclusão & Critérios de Exclusão \\
\hline $\begin{array}{l}\text { Tipo de } \\
\text { Estudos }\end{array}$ & $\begin{array}{l}\text { Revisões sistemáticas da Literatura; } \\
\text { Revisões Integrativas da Literatura; } \\
\text { Scoping review; Estudos primários } \\
\text { (quantitativos e/ou qualitativos), }\end{array}$ & $\begin{array}{l}\text { Artigos de opinião, editoriais, } \\
\text { relatos de experiência }\end{array}$ \\
\hline Participantes & $\begin{array}{l}\text { Estudantes de Enfermagem da } \\
\text { formação pré-graduada }\end{array}$ & Estudantes de mestrado e doutoramento \\
\hline $\begin{array}{l}\text { Fenómeno } \\
\text { de Interesse }\end{array}$ & $\begin{array}{l}\begin{array}{l}\text { Aprendizagem } \\
\text { envolvimento efuada pelo } \\
\text { investigação }\end{array} \\
\end{array}$ & $\begin{array}{l}\text { Aprendizagem em cursos de formação } \\
\text { sobre prática baseada em evidência ou } \\
\text { investigação, sem participação em projetos } \\
\text { 'no terreno' }\end{array}$ \\
\hline
\end{tabular}

A equação de pesquisa, efetuada entre os meses de dezembro de 2019 a fevereiro de 2020, foi (Students or undergraduate students) and (research or research projet or Technology and 
Innovation or Ativities or R\&D activities or evidence based practice) and (learning or learning outcomes) and nurs*, sendo que estes descritores também foram utilizados em língua portuguesa e inglesa e em associações (AND e OR). Para a seleção dos descritores contribuiu a análise da literatura efetuada na primeira etapa.

A pesquisa foi realizada em dois idiomas (português e inglês), nas bases de dados CINAHL®Complete; Cochrane Controlled Trials Register; Cochrane Database of Systematic Reviews; Cochrane Methodology Register; MedicLatina; MEDLINE, Scopus e JBI. Restringiu-se a pesquisa aos anos de 2014 a 2019, pela atualidade dos resultados de investigação.

O processo de seleção dos estudos foi orientado de acordo com o PRISMA (Figura1).

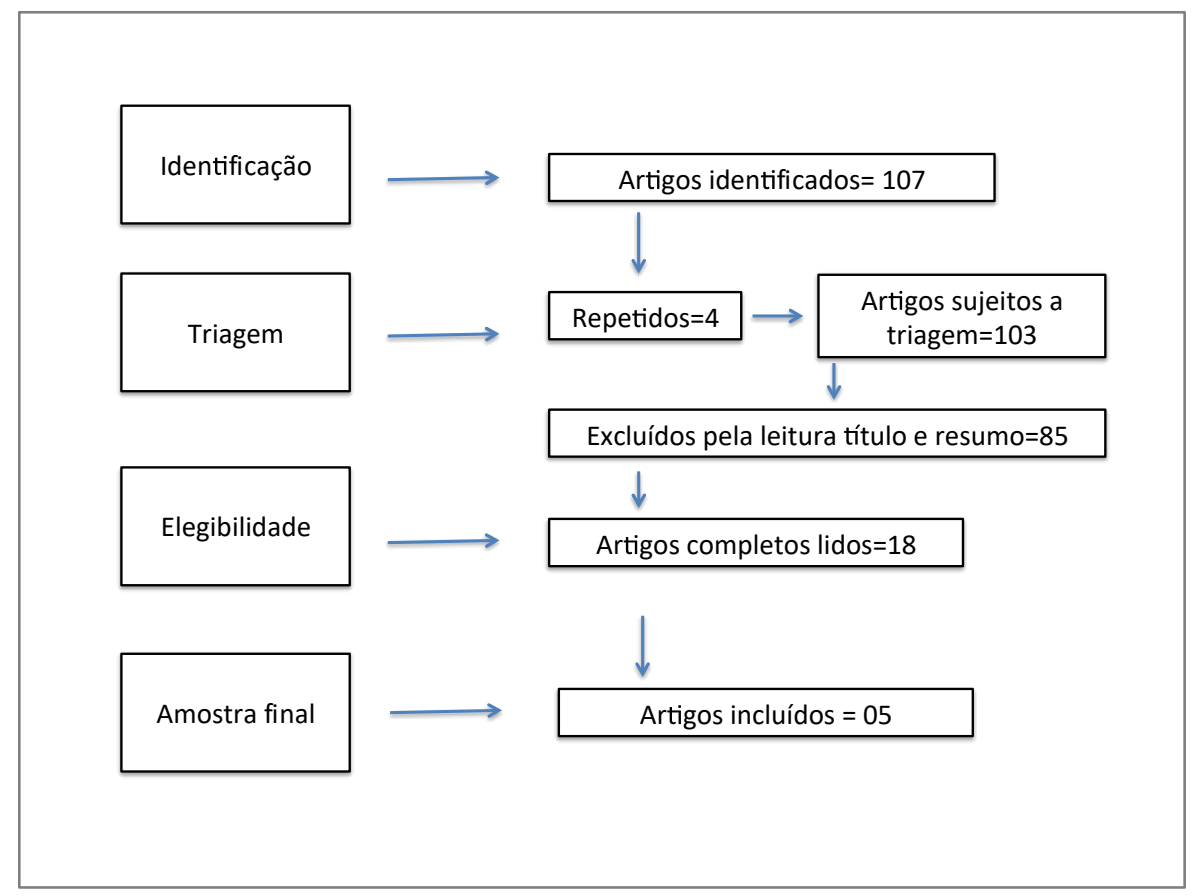

Figura 1. Fluxograma de seleção dos artigos para a revisão integrativa, elaborado a partir das recomendações PRISMA. Lisboa, 2020.

Determinou-se as informações a serem extraídas dos estudos e construiu-se um instrumento com identificação do título do artigo/obra; autor(es), ano de publicação, tipo de artigo; objetivo(s), métodos (se aplicável); avaliação do rigor metodológico; nível de evidência e principais resultados/conclusões.

A potencial amostra ficou constituída por 107 estudos que respeitavam os critérios de inclusão. A análise do título e resumo por dois revisores permitiu eliminar 89 artigos (4 
repetidos e 85 que não respeitavam os critérios de elegibilidade). Considerou-se, então, elegíveis 18 artigos. Estes foram sujeitos a análise do texto integral pelos dois investigadores, tendo-se eliminado 13 artigos que não respondiam à questão de investigação. Os artigos foram eliminados por avaliarem programas de formação em PBE ou em investigação, sem envolvimento dos estudantes em projetos de pesquisa translacional ou clínica. Identificou-se também uma Revisão Sistemática sobre o envolvimento de estudantes em investigação, mas que não permitia identificar os ganhos específicos para os estudantes de enfermagem, por isso não foi incluída na amostra (Zuchowski, Heyeres, \& Tsey, 2020).

A amostra final foi composta por 5 artigos.

\section{RESULTADOS}

Os estudos obtidos nesta RIL, e que constituem a amostra bibliográfica, são heterogéneos, quanto aos objetivos, método e resultados. Apesar da diferença das fontes e da metodologia, a sua interpretação permite dar resposta à questão de investigação. As 5 publicações têm níveis de evidência diferentes, bem como métodos e resultados distintos, conforme o descrito no Quadro 2.

Quadro 2. Artigos constituintes da amostra bibliográfica. Lisboa, 2020.

\begin{tabular}{|c|c|c|c|}
\hline $\begin{array}{l}\text { Autores (ano) } \\
\text { Pais do Estudo } \\
\text { N }\end{array}$ & $\begin{array}{l}\text { Tipo de } \\
\text { Estudo }\end{array}$ & Objetivos & Principais Resultados \\
\hline $\begin{array}{l}\text { Børsting, } \\
\text { Kristensen, \& } \\
\text { Hanssen (2020) } \\
\text { Noruega } \\
\mathrm{N}=100\end{array}$ & $\begin{array}{l}\text { Estudo } \\
\text { observacional } \\
\text { transversal }\end{array}$ & $\begin{array}{l}\text { Examinar as experiências de } \\
\text { aprendizagem de estudantes } \\
\text { da licenciatura de enfermagem, } \\
\text { depois de terem participado } \\
\text { num projeto de investigação } \\
\text { clínica. }\end{array}$ & $\begin{array}{l}\text { Os estudantes aprendem e desenvolvem } \\
\text { competências na área da comunicação, uma vez } \\
\text { que precisam de adotar diversas estratégias de } \\
\text { abordagem ao doente. } \\
\text { Desenvolvem também competências de avaliação } \\
\text { e observação clínica, de forma a completar uma } \\
\text { colheita de dados para o estudo. }\end{array}$ \\
\hline $\begin{array}{l}\text { Moskvichena, } \\
\text { Bordovskaia, \& } \\
\text { DarinsKaya (2015) } \\
\text { Rússia } \\
\mathrm{N}=40\end{array}$ & $\begin{array}{l}\text { Estudo } \\
\text { exploratório } \\
\text { Quantitativo, }\end{array}$ & $\begin{array}{l}\text { Identificar as expectativas do } \\
\text { estudantes face à investigação; } \\
\text { e comparar as estimativas com } \\
\text { a opinião dos supervisores } \\
\text { sobre as habilidades de } \\
\text { pesquisa dos alunos. }\end{array}$ & $\begin{array}{l}\text { A análise das expectativas dos alunos mostrou que } \\
\text { o apoio do supervisor é um dos fatores mais } \\
\text { importantes que motivar alunos de todos os níveis } \\
\text { de estudo a participarem de projetos de pesquisa. } \\
\text { Há ganhos no conhecimento teórico sobre os } \\
\text { fundamentos da investigação, conhecimentos e } \\
\text { habilidades de investigação (e a existência de um } \\
\text { supervisor contribui para o desenvolvimento em } \\
\text { todas as fases da investigação). }\end{array}$ \\
\hline $\begin{array}{l}\text { Jong, } \\
\text { Schout, \& Abma, } \\
(2018) \\
\text { Holanda } \\
\mathrm{N}=70\end{array}$ & $\begin{array}{l}\text { Estudo } \\
\text { retrospetivo }\end{array}$ & $\begin{array}{l}\text { Descrever a experiência em } \\
\text { envolver estudantes da } \\
\text { licenciatura em participarem } \\
\text { em atividades de investigação } \\
\text { em saúde e ilustrar o valor } \\
\text { acrescentado destas atividades } \\
\text { enquanto no papel de co- } \\
\text { investigadores. }\end{array}$ & $\begin{array}{l}\text { Os estudantes obtêm conhecimento sobre métodos } \\
\text { de investigação em enfermagem e competências } \\
\text { nesta área. } \\
\text { Motiva a autoeficácia dos estudantes na aplicação } \\
\text { destes métodos na sua prática. } \\
\text { Ajuda o estudante a transferir o conhecimento } \\
\text { teórico para a prática. }\end{array}$ \\
\hline
\end{tabular}




\begin{tabular}{|c|c|c|c|}
\hline $\begin{array}{l}\text { Autores (ano) } \\
\text { Pais do Estudo } \\
\text { N }\end{array}$ & $\begin{array}{l}\text { Tipo de } \\
\text { Estudo }\end{array}$ & Objetivos & Principais Resultados \\
\hline $\begin{array}{l}\text { Einarsen \& Giske, } \\
(2019) \\
\text { Noruega } \\
\mathrm{N}=52\end{array}$ & $\begin{array}{l}\text { Estudo } \\
\text { qualitativo } \\
\text { (análise } \\
\text { conteúdo) }\end{array}$ & $\begin{array}{l}\text { Compreender os resultados a } \\
\text { longo prazo após a } \\
\text { participação ativa em } \\
\text { pesquisas durante o segundo } \\
\text { ano do curso e a influência na } \\
\text { aprendizagem. }\end{array}$ & $\begin{array}{l}\text { Emergiram } 4 \text { categorias } \\
\text { Maior consciencialização e atenção na prática; } \\
\text { Ser construtivamente crítico com a prática clínica; } \\
\text { Maior consciencialização contextual; } \\
\text { Tornar - se um pesquisador iniciante } \\
\text { (conhecimento, entusiasmo, atitude). }\end{array}$ \\
\hline $\begin{array}{l}\text { Slattery, et al., } \\
(2016) \\
\text { EUA } \\
N=20\end{array}$ & $\begin{array}{l}\text { Estudo misto, } \\
\text { longitudinal }\end{array}$ & $\begin{array}{l}\text { Avaliar um programa de } \\
\text { envolvimento dos estudantes } \\
\text { de enfermagem } \\
\text { investigação em } \\
\text { translacional e clínica) }\end{array}$ & $\begin{array}{l}\text { A participação no programa possibilitou o } \\
\text { conhecimento dos vários papéis, } \\
\text { Aprendem a trabalhar em equipas interdisciplinares, } \\
\text { Desenvolvimento de competências de análise de } \\
\text { dados e implementação de projetos, } \\
\text { Aprofundamento do conhecimento sobre o } \\
\text { fenómeno em estudo }\end{array}$ \\
\hline
\end{tabular}

De salientar, que o estudo de Moskvichena, Bordovskaia e DarinsKaya (2015) tem uma amostra com estudantes e supervisores, sendo 40 estudantes da pré-graduação, 40 do segundo ano de mestrado e 20 de doutoramento, mas os resultados e a discussão permitem extrair os resultados de cada um dos grupos, que dão resposta à questão de investigação.

\section{DISCUSSÃO}

A amostra bibliográfica deste estudo é constituída por 5 artigos que obedecem aos critérios de inclusão pré-estabelecidos no protocolo e que permitem dar resposta à questão de investigação.

As investigações são heterogéneas do ponto de vista do desenho do estudo e em termos de tamanho e características da amostra, medidas e momentos de avaliação da efetividade da intervenção (momento em que foram envolvidos) o que dificulta a comparação dos resultados. Os estudos são todos originais $(n=5)$ e predomina a investigação quantitativa $(n=4)$.

Os resultados desta RIL ilustram os benefícios do envolvimento dos estudantes de enfermagem em projetos de investigação. Independentemente da metodologia e da fase do processo de pesquisa e que pode incluir atividades diferentes, desde a participação na formulação da questão de pesquisa, à revisão de literatura, ao desenho do estudo, à colheita e análise de dados, até à divulgação dos resultados com comunicações e participação na redação de artigos científicos, há ganhos em termos de conhecimento e motivação, até pela constatação que a concretização das atividades que the são solicitadas 
ajuda no progresso da investigação (Slattery et al., 2016; Jong et al., 2018; Einarsen \& Giske, 2019; Børsting, Kristensen, \& Hanssen, 2020).

Estes resultados são subsidiados pela opinião de vários autores que recomendam o envolvimento dos estudantes em projetos de investigação como uma estratégia importante para a aquisição e conhecimento e desenvolvimento de competências para adesão a uma verdadeira PBE, enquanto futuros profissionais (Jansen et al., 2015; Long, Bischoff, \& Aduddell, 2018; Oh \& Yang, 2019; Mendes et al., 2019; Zuchowski, Heyeres, \& Tsey, 2020) e pela oportunidade de contribuir para o desenvolvimento do saber na área da profissão que escolheram, enquanto dão resposta aos resultados de aprendizagem esperados para a licenciatura (Jansen et al., 2015).

Os resultados do estudo de Slattery et al. (2016) salientam que a participação no programa possibilitou o conhecimento dos vários papéis e o desenvolvimento de capacidade para trabalhar em equipas interdisciplinares. O que reforça a constatação Jansen et al. (2015) de que os alunos têm, nestas atividades, oportunidades de liderança, de promoção do trabalho em equipe interdisciplinar e ajudar a universidade a alcançar objetivos estratégicos. Estas experiências auxiliam os alunos a perceberem o seu envolvimento como uma oportunidade de entrar em contato com pesquisa e inovação, desenvolver competências e interagir com outras pessoas (Mendes et al., 2019), o que os leva a descrever as oportunidades como desafiantes e interessantes (Mendes et al., 2019; Oh \& Yang, 2019).

Os benefícios também incluem a criação de experiências eficazes de aprendizagem que constroem o conhecimento de enfermagem e potencialmente contribuem para a saúde da comunidade (Jansen et al., 2015; Jong, Meijer, Schout, \& Abma, 2018; Long, Bischoff, \& Aduddell, 2018).

Os dados da pesquisa de Moskvichena, Bordovskaia, \& Darinskaya (2015) reportam para o papel do supervisor como central para a orientação, aprendizagem e motivação dos 'investigadores juniores'. Por outro lado, o contacto com os orientadores e a sua experiência, em situações que envolvem a resolução de problemas e tomada de decisão complexa possibilita não só a aprendizagem da evidência, mas também a transferência do conhecimento e o desenvolvimento de habilidades e competências transversais necessárias para a mudança na profissão de enfermagem (Baixinho et al., 2017; Jong et al., 2018). 
Apesar do objetivo desta RIL não ser a avaliação de programas de formação em PBE, importa, mesmo assim, referir os resultados do estudo de Kim, Gu e Chang, (2019) que desenvolveram um programa de educação em PBE para estudantes de enfermagem e avaliaram o efeito do programa no conhecimento, capacidades, atitudes, competências e utilização futura da evidência. Concluíram que o grupo experimental apresentou scores pósteste, estatisticamente mais altos, no conhecimento da PBE $(p<0,001)$, habilidades ( $p$ $<0,001$ ), atitudes $(p<0,001)$, competências $(p<0,001)$, uso futuro da PBE $(p=0,001)$ e pensamento crítico $(p<0,001)$, comparado ao grupo controle. Estes dados justificam que estudos futuros explorem a associação entre a formação e a participação em estudos de investigação e o impacto nestas variáveis.

Os achados desta revisão permitem corroborar a opinião de Ommering et al. (2019) que em relação à educação médica defendem a participação de estudantes em pesquisa durante a faculdade de medicina porque contribui para criar uma atitude académica, subjacente à prática da medicina baseada em evidência.

Convém realçar que as experiências de pesquisa são particularmente adequadas para criar aprendizagens quando ocorrem em ambientes de prática real focados em problemas clinicamente relevantes (Oh \& Yang, 2019; Børsting, Kristensen, \& Hanssen, 2020).

A investigação é uma competência essencial para os profissionais de saúde e implica que estejam cientes dos mais recentes desenvolvimentos na área da saúde, sejam capazes de avaliar criticamente a literatura científica e utilizar o conhecimento científico na tomada de decisões clínicas (Oh \& Yang, 2019; Ommering et al., 2019). Por isso, pesquisar durante a licenciatura é um foco emergente para ajudar os alunos a desenvolver as habilidades e competências necessárias para um profissional de saúde na atualidade (Long, Bischoff, \& Aduddell, 2018; Zuchowski, Heyeres, \& Tsey, 2020).

Apesar de existirem inúmeras vantagens já descritas, e o consenso da literatura quanto à importância do envolvimento em atividades de investigação por parte de estudantes da licenciatura, existem algumas barreiras como a falta de conhecimento e/ou atitudes negativas sobre a investigação e o envolvimento de estudantes na mesma, dificuldades na comunicação, divulgação e apropriação dos resultados pelos profissionais, falta de apoio institucional e económico necessário para realizar pesquisas (Long, Bischoff, \& Aduddell, 2018). Jansen et al. (2015) consideram que o grande desafio no envolvimento dos 
estudantes é o tempo necessário para orientar e supervisionar os alunos, porque eles precisam de aprender e de supervisão (Jansen et al., 2015).

Assiste-se neste momento a uma mudança paradigmática, com um apelo pronunciado à transição da educação informada por pesquisa, na qual os estudantes são consumidores passivos de conhecimento, para a educação baseada em pesquisa, na qual os alunos estão realmente envolvidos nos estudos, assimilando, assim, o conhecimento de uma forma ativa (Ommering et al., 2019).

Não havendo indicações precisas do momento ideal para envolver os estudantes, é interessante a ideia de Ommering et al., (2019) ao observar que "a prática leva à perfeição", sendo, por isto, importante envolver os alunos na pesquisa o mais cedo possível.

\section{CONCLUSÕES}

Os resultados desta revisão integrativa da literatura mostram que a participação de estudantes da licenciatura em enfermagem em projetos de investigação contribui para diferentes aprendizagens, desenvolvimento de atitudes e competências científicas.

Os estudantes podem ser envolvidos em todas as fases do processo de pesquisa, com benefícios para a sua aprendizagem, motivação e para o próprio desenvolvimento da investigação. Esta prática pedagógica possibilita aprendizagem, reflexão e integração do conhecimento, desenvolvimento de competências de comunicação, escrita científica, gestão do tempo, juízo critico e aumenta a satisfação com o processo de aprendizagem.

Os resultados confirmam a afirmação de alguns autores, fundamentando a ideia de que a PBE desenvolve-se pela participação dos futuros profissionais em projetos de investigação, aumentando a sua capacidade de leitura, produção e síntese de conhecimento. Por isso, a formação teórica sobre investigação deve ser associada a outras estratégias para o desenvolvimento de conhecimentos, habilidades e atitudes científicas.

As limitações do estudo são decorrentes da dimensão da amostra bibliográfica, da heterogeneidade dos estudos e da própria natureza da experiência de investigação que o estudante teve possibilidade de realizar. A pesquisa incluiu estudos em português e inglês, não identificando estudos publicados em outras línguas e não tendo sido incluída literatura cinzenta, pelo que poder-se-ão ter perdido alguns estudos de mestrado e doutoramento sobre o tema. 
A não identificação de estudos nacionais sobre o tema e o tamanho da amostra $(n=5)$ justificam a necessidade de estudos em Portugal sobre o tema. Os resultados permitem sugerir que os estudos futuros devem explorar a participação dos estudantes em projetos de investigação e a relação com a literacia científica e as atitudes face à PBE no contexto de cuidados de saúde.

\section{REFERÊNCIAS}

Aglen, B. (2016). Pedagogical strategies to teach bachelor students evidence-based practice: A systematic review. Nurse Educ Today, 36, 255-63. doi: 10.1016/j. nedt.2015.08.025

Allari, R. S. (2016). Students' Experiences in Nursing Research and Evidence based Practice Course: A Qualitative Study. Int J Nurs. 3(2), 62-8. doi: 10.15640/ijn.v3n2a9

Baixinho, C. L., Ferreira, Ó., Marques, F. M., Presado, M. H., \& Cardoso, M. (2017). Transição segura: um projeto da transferência do conhecimento para a prática clínica. In Costa A. P., Sánches-Gómez M. C., Cilleros M.V.M. A prática na Investigação Qualitativa: exemplos de estudos. Oliveira de Azeméis: Ludomédia, pp.57-80.

Børsting T. E., Kristensen, N., \& Hanssen, I. (2020). Student nurses' learning outcomes through participation in a clínical nursing research project: A qualitative study, Nurs Educ Practice, 43, 102727. doi: https://doi.org/10.1016/j.nepr.2020.102727.

Cardoso, D. F. B., Coelho, A. R. N., Louçano, C. C., Parola, V. S. O., Rodrigues, M. A., Fineout-Overholt, E. \& Apóstolo, J. L. A. (2019). Translation and cross-cultural adaptation of evidence-based practice instruments for Portuguese nursing students. Rev Enf Referência, IV(23), 141-21. doi: https://doi.org/10.12707/RIV19058

Einarsen, K. A., \& Giske, T. (2019). Nursing students' longitudinal learning outcomes after participation in a research project in a hospital. IPDJ., 9(1), 1-4. doi: https://doi.org/10.19043/ipdj.91.004

Foster, J., \& Flanders, S. (2014). Challenges in clínical nurse specialist education and practice. Online J Issues Nurs., $19(2), 1$. doi:10.3912/OJIN.Vol19No02Man01

Jansen, D. A., Jadack, R. A., Ayoola, A. B., Doornbos, M. M., Dunn, S. L, ... Wegner, G. D. (2015). Embedding Research in Undergraduate Learning Opportunities. West J Nurs Res., 37(10), 1340-58. doi: 10.1177/0193945915571136. Epub 2015 Feb 17.

Jong, G., Meijer, E., Schout, G., \& Abma, T. (2018). Involving Undergraduate Nursing Students in Participatory Health Research: Implications from the Netherlands. J Prof Nurs; 34(6): 507-513. doi: https://doi.org/10.1016/j.profnurs.2018.04.001.

Kim, J. S., Gu, M. O. \& Chang, H. (2019). Effects of an evidence-based practice education program using multifaceted interventions: a quasi-experimental study with undergraduate nursing students. BMC Med Educ., 19, 71. doi: https://doi.org/10.1186/s12909-019-1501-6

Long, A., Bischoff, W. R., \& Aduddell, K. (2018). Research Prescription for Undergraduate Students: Research Mentoring in a Small Liberal Arts University. J Prof Nurs., 35(3), 170-3. doi: https://doi.org/10.1016/j.profnurs.2018.12.007

Mena-Tudela, D., González-Chordá, V. M., Cervera-Gasch, A., Maciá-Soler, M. L., \& Orts-Cortés, M. I. (2018). Effectiveness of an Evidence-Based Practice educational intervention with second-year nursing students. Rev Latino-Am Enfermagem, 26, e3026. doi:https://dx.doi.org/10.1590/1518-8345.2502.3026

Mendes, A., Silva, I. C., Henriques, A., Claúdio, A. P., Balsa, J., Carmos, M. B. ... Guerreiro, M. (2019). Involving undergraduate nursing students in a multidisciplinary research project: strategy for implementation, first results and future perspectives. Ann. Med., 51(sup1), 205. doi: 10.1080/07853890.2018.1560165

Mendes, K. D. S., Silveira, R. C. C. P., \& Galvão, C. M. (2008). Revisão integrativa: método de pesquisa para a incorporação de evidências na saúde e na enfermagem. Texto Contexto Enferm., 17(4), 758-64. doi: http://dx.doi.org/10.1590/S010407072008000400018. 
Moskvichena, N., Bordovskaia, N., \& Darinskaya, L. (2015). Role of Students and Supervisors' Interaction in Research Projects: Expectations and Evaluations. Procedia Soc Behav Sci., 171(2015), 576-83. doi: https://doi.org/10.1016/j.sbspro.2015.01.163

Oh, E. G., \& Yang, Y. L. (2019). Evidence-based nursing education for undergraduate students: A preliminary experimental study. Nurse Educ Pract., 38, 45-51. doi: 10.1016/j.nepr.2019.05.010. Epub 2019 May 31.

Ommering, B. W. C., Diepen, M. V., van Blankenstein, F. M., Jong, P. G. M., \& Dekker, F. W. (2019). Twelve tips to offer a short authentic and experiential individual research opportunity to a large group of undergraduate students. Med Teach. doi: 10.1080/0142159X.2019.1695766

Ross, J. G., \& Burrell, S. A. (2019). Nursing students' attitudes toward research: An integrative review. Nurs Educ Today, 82 , 79-87. doi: https://doi.org/10.1016/j.nedt.2019.08.006.

Slattery, M. J., Logan, B. L., Mudge, B., Secore, K., von Reyn, L. J., \& Maue, R. A. (2016). An Undergraduate Research Fellowship Program to Prepare Nursing Students for Future Workforce Roles. J Prof Nurs; 32(6), 412-420. doi: https://doi.org/10.1016/j.profnurs.2016.03.008

Sun, J. H., Liu, J. E., Wu, Y., \& Li, S. J. (2014). The Effects Of The Student-Centered Clínical Nursing Practice Mode Based On The Action Research For Clínical Practicum Of Undergraduate Students In Beijing, China. Procedia Soc Behav Sci; 141(2014), 839-45. doi: 10.1016/j.sbspro.2014.05.146

Zuchowski, I., Heyeres, M. \& Tsey, K. (2020). Students in Research Placements as Part of Professional Degrees: A Systematic Review. Aus. Soc. Work., 73(1), 48-63. doi: 10.1080/0312407X.2019.1649439 